\title{
Primitive minima of positive definite quadratic forms
}

\author{
by
}

\author{
Aloys KriEg (Münster)
}

1. Introduction. The main purpose of the reduction theory is to construct a fundamental domain of the unimodular group acting discontinuously on the space of positive definite quadratic forms. This fundamental domain is for example used in the theory of automorphic forms for $\mathrm{GL}_{n}$ (cf. [11]) or in the theory of Siegel modular forms (cf. [1], [4]). There are several ways of reduction, which are usually based on various minima of the quadratic form, e.g. the Korkin-Zolotarev method (cf. [10], [3]), Venkov's method (cf. [12]) or Voronoi's approach (cf. [13]), which also works in the general setting of positivity domains (cf. [5]). The most popular method is Minkowski's reduction theory [6] and its generalizations (cf. [9], [15]).

Minkowski's reduction theory is based on attaining certain minima, which can be characterized as the successive primitive minima of the quadratic form. Besides these we have successive minima, but a reduction according to successive minima only works for $n \leq 4$ (cf. [14]). In this paper we introduce so-called primitive minima, which lie between successive and successive primitive minima (cf. Theorem 2). Using primitive minima we obtain a straightforward generalization of Hermite's inequality in Theorem 1. As an application we get a simple proof for the finiteness of the class number. Finally we describe relations with Rankin's minima (cf. [8]) and with Venkov's reduction (cf. [12]).

2. Various minima. Let $\mathcal{P}_{n}$ denote the set of all real positive definite $n \times n$ matrices. $\mathrm{GL}_{n}(\mathbb{Z})$ stands for the unimodular group of degree $n$, i.e. the group of units in the ring $M_{n}(\mathbb{Z})$. An integral $n \times k$ matrix $P \in M_{n \times k}(\mathbb{Z})$, $n \geq k$, is called primitive, if the g.c.d. of all the $k$-rowed minors of $P$ is 1 . This is equivalent to the fact that there exists a matrix $(P, *) \in \mathrm{GL}_{n}(\mathbb{Z})$ (cf. [7]). Moreover, set

$$
A[B]:=\left(B^{t}\right) A B
$$

for matrices $A, B$ of appropriate size. 
A matrix $S=\left(s_{j k}\right) \in \mathcal{P}_{n}$ is called Minkowski-reduced whenever

$$
\begin{aligned}
& \text { (M.1) } S[g] \geq s_{k k} \text { for all } g=(\left(\gamma_{1}, \ldots, \gamma_{n}\right)^{t} \in \mathbb{Z}^{n} \\
& \text { with g.c.d. }\left(\gamma_{k}, \ldots, \gamma_{n}\right)=1,1 \leq k \leq n, \\
& \text { (M.2) } \quad s_{k, k+1} \geq 0 \text { for } 0<k<n .
\end{aligned}
$$

The set of Minkowski-reduced matrices is a fundamental domain of $\mathcal{P}_{n}$ with respect to the discontinuous group of mappings

$$
\mathcal{P}_{n} \rightarrow \mathcal{P}_{n}, \quad S \mapsto S[U], \quad U \in \mathrm{GL}_{n}(\mathbb{Z}) .
$$

In order to determine a unimodular matrix $U$ such that $S[U]$ is Minkowskireduced proceed as follows (cf. [4]): Given $S \in \mathcal{P}_{n}$ define its minimum by

$$
\mu(S):=\inf \left\{S[h] \mid 0 \neq h \in \mathbb{Z}^{n}\right\} .
$$

Determine $g_{1} \in \mathbb{Z}^{n}$ with $\mu(S)=S\left[g_{1}\right]$. As soon as $g_{1}, \ldots, g_{k}, 0<k<n$, are given, choose $g_{k+1} \in \mathbb{Z}^{n}$ such that

$$
S\left[g_{k+1}\right]=\inf \left\{S[h] \mid\left(g_{1}, \ldots, g_{k}, h\right) \in M_{n \times(k+1)}(\mathbb{Z}) \text { is primitive }\right\} .
$$

If necessary replace $g_{k+1}$ by $-g_{k+1}$ in order to get $g_{k} S g_{k+1} \geq 0$. In this way we construct a unimodular matrix $U=\left(g_{1}, \ldots, g_{n}\right)$ such that $T=S[U]$ is Minkowski-reduced. The diagonal entries of $T$ are given by (1) and (2) and may therefore be called the successive primitive minima of $S$.

Besides these the successive minima $\mu_{1}(S), \ldots, \mu_{n}(S)$ of $S \in \mathcal{P}_{n}$ were introduced (cf. [14]). Determine $g_{1} \in \mathbb{Z}^{n}$ as in (1), i.e.

$$
\mu_{1}(S)=\mu(S)=S\left[g_{1}\right] .
$$

As soon as $g_{1}, \ldots, g_{k}, 0<k<n$, are given, choose $g_{k+1} \in \mathbb{Z}^{n}$ such that

(3) $\mu_{k+1}(S)=S\left[g_{k+1}\right]=\inf \left\{S[h] \mid h \in \mathbb{Z}^{n}, \operatorname{rank}\left(g_{1}, \ldots, g_{k}, h\right)=k+1\right\}$.

Using Steinitz' theorem we have the alternative definition

(4) $\mu_{k}(S)=\inf \left\{\begin{array}{l|l}t \in \mathbb{R} & \begin{array}{l}\text { there is } H=\left(h_{1}, \ldots, h_{k}\right) \in M_{n \times k}(\mathbb{Z}), \\ \operatorname{rank} H=k, S\left[h_{j}\right] \leq t, 1 \leq j \leq k\end{array}\end{array}\right\}$,

$$
1 \leq k \leq n \text {. }
$$

Comparing (3) and (4) it is interesting to investigate the analogue for primitive matrices in place of maximal rank matrices. We define

$$
\begin{aligned}
& \nu_{k}(S)=\inf \left\{\begin{array}{l|l}
t \in \mathbb{R} & \begin{array}{l}
\text { there is a primitive } H=\left(h_{1}, \ldots, h_{k}\right) \\
\text { in } M_{n \times k}(\mathbb{Z}), S\left[h_{j}\right] \leq t, 1 \leq j \leq k
\end{array}
\end{array}\right\}, \\
& 1 \leq k \leq n .
\end{aligned}
$$

We call $\nu_{k}(S)$ the $k$-th primitive minimum of $S$. Obviously one has

$$
\mu_{k}(S) \leq \nu_{k}(S), \quad 1 \leq k \leq n, \quad \nu_{1}(S)=\mu_{1}(S)=\mu(S) .
$$


3. A generalization of Hermite's inequality. For $S \in \mathcal{P}_{n}$ we have

$$
\mu(S)=\nu_{1}(S) \leq \nu_{2}(S) \leq \ldots \leq \nu_{n}(S) .
$$

Since $U P, U \in \mathrm{GL}_{n}(\mathbb{Z})$, is primitive if and only if $P$ is, we conclude

$$
\nu_{k}(S[U])=\nu_{k}(S) \quad \text { for } U \in \mathrm{GL}_{n}(\mathbb{Z}), 1 \leq k \leq n .
$$

Note that a primitive matrix can be completed to a unimodular matrix. Hence given $1 \leq k \leq n$ there exists $U_{k} \in \mathrm{GL}_{n}(\mathbb{Z})$ such that

$$
S\left[U_{k}\right]=T=\left(t_{i j}\right), \quad t_{11} \leq t_{22} \leq \ldots \leq t_{n n}, \quad t_{k k}=\nu_{k}(S) .
$$

Theorem 1. Given $S \in \mathcal{P}_{n}$ one has

$$
\nu_{1}(S) \ldots \nu_{n}(S) \leq\left(\frac{4}{3}\right)^{n(n-1) / 2} \operatorname{det} S .
$$

Proof. We use induction on $n$; the case $n=1$ is obvious. According to (8) and (9) we may assume $s_{11}=\mu(S)=\nu_{1}(S)=: \mu$ without restriction. By the method of completing squares we obtain a decomposition

$$
S=\left(\begin{array}{cc}
\mu & 0 \\
0 & T
\end{array}\right)\left[\left(\begin{array}{cc}
1 & a^{t} \\
0 & I
\end{array}\right)\right]=\left(\begin{array}{cc}
\mu & \mu a^{t} \\
\mu a & T+\mu a a^{t}
\end{array}\right), \quad T \in \mathcal{P}_{n-1}, a \in \mathbb{R}^{n-1},
$$

where $I$ is the $(n-1) \times(n-1)$ identity matrix. Given $0<k<n$ there exists a primitive matrix $G=\left(g_{1}, \ldots, g_{k}\right) \in M_{(n-1) \times k}(\mathbb{Z})$ such that

$$
T\left[g_{j}\right] \leq \nu_{k}(T), \quad 1 \leq j \leq k .
$$

Next choose $g=\left(\gamma_{1}, \ldots, \gamma_{k}\right)^{t} \in \mathbb{Z}^{k}$ such that the entries of $g+G^{t} a$ belong to the interval $\left[-\frac{1}{2} ; \frac{1}{2}\right]$. Now

$$
H=\left(\begin{array}{ll}
1 & g^{t} \\
0 & G
\end{array}\right) \in M_{n \times(k+1)}(\mathbb{Z}) \quad \text { and } \quad H^{\prime}=\left(\begin{array}{l}
g^{t} \\
G
\end{array}\right) \in M_{n \times k}(\mathbb{Z})
$$

are primitive. One has

$$
S\left[\begin{array}{l}
\gamma_{j} \\
g_{j}
\end{array}\right]=\mu\left(\gamma_{j}+a^{t} g_{j}\right)^{2}+T\left[g_{j}\right] \leq \frac{1}{4} \nu_{1}(S)+\nu_{k}(T), \quad 1 \leq j \leq k .
$$

Since $H^{\prime}$ is primitive we conclude

$$
\nu_{k}(S) \leq \frac{1}{4} \nu_{1}(S)+\nu_{k}(T) .
$$

Now (7) leads to

$$
S\left[\begin{array}{l}
1 \\
0
\end{array}\right]=\nu_{1}(S) \leq \nu_{k}(S) \leq \frac{1}{4} \nu_{1}(S)+\nu_{k}(T) .
$$

Since $H$ is primitive, we now have

$$
\nu_{k+1}(S) \leq \frac{1}{4} \nu_{1}(S)+\nu_{k}(T) \quad \text { and } \quad \nu_{k+1}(S) \leq \frac{4}{3} \nu_{k}(T) .
$$

According to $\nu_{1}(S) \operatorname{det} T=\operatorname{det} S$ the induction hypothesis yields

$$
\begin{aligned}
\nu_{1}(S) \ldots \nu_{n}(S) & \leq\left(\frac{4}{3}\right)^{n-1} \nu_{1}(S) \nu_{1}(T) \ldots \nu_{n-1}(T) \\
& \leq\left(\frac{4}{3}\right)^{n(n-1) / 2} \nu_{1}(S) \operatorname{det} T=\left(\frac{4}{3}\right)^{n(n-1) / 2} \operatorname{det} S .
\end{aligned}
$$


In view of (7) we obtain Hermite's inequality (cf. [7]) as

Corollary 1. Given $S \in \mathcal{P}_{n}$ one has

$$
\mu(S)^{n} \leq\left(\frac{4}{3}\right)^{n(n-1) / 2} \operatorname{det} S .
$$

Denote the class number by $h_{n}(N), N \geq 1$, i.e. $h_{n}(N)$ is the number of $\mathrm{GL}_{n}(\mathbb{Z})$-equivalence classes of integral $S \in \mathcal{P}_{n}$ with $\operatorname{det} S=N$.

Corollary 2. The class numbers $h_{n}(N), N \geq 1$, are finite. One has

$$
h_{n}(N)=O\left(N^{n(n+1) / 2}\right) \quad \text { as } N \rightarrow \infty .
$$

Proof. By (9) it suffices to count the number of integral $S \in \mathcal{P}_{n}$ with $\operatorname{det} S=N$ and $s_{k k} \leq \nu_{n}(S), 1 \leq k \leq n$. In view of $\nu_{k}(S) \geq 1$ Theorem 1 implies

$$
0<s_{k k} \leq \nu_{n}(S) \leq \nu_{1}(S) \ldots \nu_{n}(S) \leq\left(\frac{4}{3}\right)^{n(n-1) / 2} N .
$$

Next $S \in \mathcal{P}_{n}$ yields $s_{j j} s_{k k}-s_{j k}^{2}>0$, hence $\left|s_{j k}\right|<\left(\frac{4}{3}\right)^{n(n-1) / 2} N$ for $1 \leq$ $j<k \leq n$. Thus the number of these $S$ is $O\left(N^{n(n+1) / 2}\right)$ as $N \rightarrow \infty$.

For other proofs of Corollary 2 we refer to [7].

4. Relations with other types of minima. The first relation is derived in

Theorem 2. Let $S=\left(s_{i j}\right) \in \mathcal{P}_{n}$ be Minkowski-reduced. Given $1 \leq k \leq n$ one has

$$
\mu_{k}(S) \leq \nu_{k}(S) \leq s_{k k} \leq \alpha_{k} \mu_{k}(S) \leq \alpha_{k} \nu_{k}(S)
$$

where

$$
\alpha_{k}= \begin{cases}1 & \text { if } k \leq 4 \\ \left(\frac{5}{4}\right)^{k-4} & \text { if } k \geq 4 .\end{cases}
$$

Proof. $\nu_{k}(S) \leq s_{k k}$ follows from $s_{11} \leq \ldots \leq s_{n n}$. The remaining parts are consequences of (6) and [14], Satz 7 and (45).

If $k \geq 5$ there are quadratic forms $S$ with $\nu_{k}(S)>\mu_{k}(S)$. Just as in [14] consider the matrix $S$ attached to the quadratic form

$$
x_{1}^{2}+x_{2}^{2}+x_{3}^{2}+x_{4}^{2}+\left(x_{1}+x_{2}+x_{3}+x_{4}\right) x_{5}+\frac{5}{4} x_{5}^{2} .
$$

One easily checks

$$
\mu_{k}(S)=\nu_{j}(S)=1, \quad 1 \leq k \leq 5,1 \leq j \leq 4, \quad \nu_{5}(S)=\frac{5}{4} .
$$

Next consider the minima

$$
\begin{aligned}
\delta_{k}(S) & :=\inf \left\{\operatorname{det}(S[P]) \mid P \in M_{n \times k}(\mathbb{Z}) \text { primitive }\right\} \\
& =\inf \left\{\operatorname{det}(S[G]) \mid G \in M_{n \times k}, \operatorname{rank} G=k\right\}, \quad 1 \leq k \leq n,
\end{aligned}
$$

which were introduced by Rankin [8]. 
Proposition 1. Given $S \in \mathcal{P}_{n}$ and $1 \leq k \leq n$ one has

$$
\nu_{1}(S) \ldots \nu_{k}(S) \leq\left(\frac{4}{3}\right)^{k(k-1) / 2} \delta_{k}(S) .
$$

Proof. Choose a primitive $P \in M_{n \times k}(\mathbb{Z})$ with $\delta_{k}(S)=\operatorname{det}(S[P])$. Apply Theorem 1 to $S[P]$. In view of the obvious inequalities $\nu_{j}(S[P]) \geq$ $\nu_{j}(S)$ for $1 \leq j \leq k$, the claim follows.

Given $T \in \mathcal{P}_{k}$ and $S \in \mathcal{P}_{n}, 1 \leq k \leq n$, we define

$$
\nu_{T}(S):=\inf \left\{\operatorname{tr}(S[P] T) \mid P \in M_{n \times k}(\mathbb{Z}) \text { primitive }\right\},
$$

where $t r$ is the trace. Clearly the minimum is attained and one has

$$
\nu_{I}(S) \geq \nu_{1}(S)+\ldots+\nu_{k}(S), \quad I=\left(\begin{array}{lll}
1 & & 0 \\
& \ddots & \\
0 & & 1
\end{array}\right) \in \mathcal{P}_{k},
$$

where equality holds at least for $k \leq 4$. If $k=n$ and $T \in \mathcal{P}_{n}$ has no non-trivial automorphs, then Venkov [12] showed that

$$
\left\{S \in \mathcal{P}_{n} \mid \operatorname{tr}(S T)=\nu_{T}(S)\right\}
$$

is a fundamental domain of $\mathcal{P}_{n}$ with respect to the action of the unimodular group.

Proposition 2. Let $S \in \mathcal{P}_{n}, T \in \mathcal{P}_{k}, 1 \leq k \leq n$. Then one has

$$
\nu_{T}(S) \geq k \delta_{k}(S)^{1 / k}(\operatorname{det} T)^{1 / k} \geq k\left(\frac{3}{4}\right)^{(k-1) / 2} \mu(S) \mu(T) .
$$

Proof. Choose a primitive $P \in M_{n \times k}(\mathbb{Z})$ with $\nu_{T}(S)=\operatorname{tr}(S[P] T)$. Then apply the result of Barnes and Cohn [2] to $S[P]$ and $T$ :

$$
\nu_{T}(S)=\operatorname{tr}(S[P] T) \geq k(\operatorname{det}(S[P]))^{1 / k}(\operatorname{det} T)^{1 / k} .
$$

One has $\operatorname{det}(S[P]) \geq \delta_{k}(S)$. Now the claim follows by virtue of Proposition 1, Corollary 1 and (7).

\section{References}

[1] A. N. Andrianov, Quadratic Forms and Hecke Operators, Grundlehren Math. Wiss. 286, Springer, Berlin 1987.

[2] E. S. Barnes and M. J. Cohn, On the inner product of positive quadratic forms, J. London Math. Soc. (2) 12 (1975), 32-36.

[3] D. Grenier, Fundamental domains for the general linear group, Pacific J. Math. 132 (1988), 293-317.

[4] H. Klingen, Introductory Lectures on Siegel Modular Forms, Cambridge University Press, Cambridge 1990.

[5] M. Koecher, Beiträge zu einer Reduktionstheorie in Positivitätsbereichen I, Math. Ann. 141 (1960), 384-432.

[6] H. Minkowski, Diskontinuitätsbereich für arithmetische Äquivalenz, J. Reine Angew. Math. 129 (1905), 220-274. 
[7] M. Newman, Integral Matrices, Academic Press, New York 1972.

[8] R. A. Rankin, On positive definite quadratic forms, J. London Math. Soc. 28 (1953), 309-319.

[9] S. S. Ryshkov, On the Hermite-Minkowski reduction theory for positive quadratic forms, J. Soviet Math. 6 (1976), 651-671.

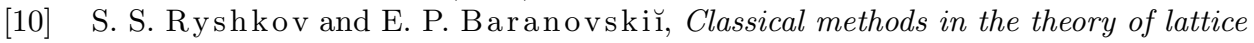
packings, Russian Math. Surveys 34 (4) (1979), 1-68.

[11] A. Terras, Harmonic Analysis on Symmetric Spaces and Applications II, Springer, New York 1988.

[12] A. B. Venkov, On the reduction of positive quadratic forms, Izv. Akad. Nauk SSSR Ser. Mat. 4 (1940), 37-52 (in Russian).

[13] G. Voronoï, Sur quelques propriétés des formes quadratiques positives parfaites, J. Reine Angew. Math. 133 (1907), 97-178.

[14] B. L. van der Waerden, Die Reduktionstheorie der positiven quadratischen Formen, Acta Math. 96 (1956), 263-309.

[15] H. Weyl, Theory of reduction for arithmetical equivalence, Trans. Amer. Math. Soc. 48 (1940), 126-164.

MATHEMATISCHES INSTITUT

WESTFÄLISCHE WILHELMS-UNIVERSITÄT

EINSTEINSTR. 62

W-4400 MÜNSTER

FEDERAL REPUBLIC OF GERMANY

E-mail: ALOYS@MATH.UNI-MUENSTER.DE 